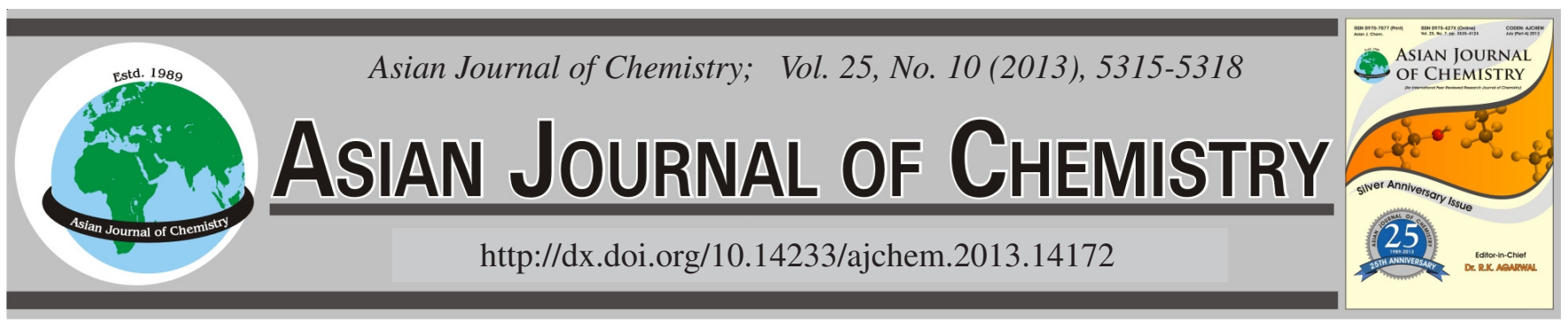

\title{
Electrochemical Determination of Ranitidine Hydrochloride in Pharmaceutical Formulations and Biological Fluids at Graphene Modified Electrode
}

\author{
XIA XI and Liang Ming*
}

School of Chemistry and Chemical Engineering, Nantong University, Nantong 226007, Jiangsu Province, P.R. China

*Corresponding author: E-mail: nt_mingliang@163.com

\begin{abstract}
A simple and sensitive method is described for electrochemical determination of ranitidine hydrochloride, a widely used histamine $\mathrm{H}_{2}$-receptor antagonist, based on its electrochemical oxidation at a graphene modified glassy carbon electrode (GCE). Under optimized conditions, the anodic peak current was linear to the concentration of ranitidine hydrochloride in the range from $3.0 \times 10^{-7}-1.0 \times 10^{-4} \mathrm{~mol} / \mathrm{L}$ with the detection limit of $1.0 \times 10^{-7} \mathrm{~mol} / \mathrm{L}$. The relative standard deviation (RSD) was $2.6 \%$ for $2.0 \times 10^{-5} \mathrm{~mol} / \mathrm{L}$ ranitidine hydrochloride $(\mathrm{n}=10)$. To further validate its possible application, the proposed method was successfully used for the determination of ranitidine hydrochloride in pharmaceutical formulations and biological fluids with satisfactory results.
\end{abstract}

Key Words: Ranitidine hydrochloride, Electrochemical determination, Graphene, Modified electrode.

ᄂ - - - - - - - - - - - - - - - - - - - - - - - - - - -

\section{INTRODUCTION}

Ranitidine hydrochloride (RNH), chemically N-[2-[[[5[(dimethylamino)methyl]-2-furanyl] methyl]thio]ethyl]-N'methyl-2-nitro-1,1-ethenediamine hydrochloride (Fig. 1), is a histamine $\mathrm{H}_{2}$-receptor antagonist widely used in the treatment of duodenal and gastric ulceration, gastroesophageal reflux disease and conditions of elevated gastric acidity, such as Zollinger-Ellison syndrome ${ }^{1}$. However, massive overdose of ranitidine hydrochloride may cause side effects such as severe hepatotoxicity, hepatitis and disorder in visual accommodation ${ }^{2}$. Therefore, the determination of ranitidine hydrochloride is of great importance in biological fluids and pharmaceutical formulations analysis. Numerous analytical techniques have been reported for the determination of ranitidine hydrochloride, which include high performance liquid chromatography $^{3,4}$, high pressure thin layer chromatography ${ }^{5}$, fluorometry ${ }^{6}$, spectrophotometry ${ }^{7}$, titrimetry ${ }^{8}$, flow-injection chemiluminescence ${ }^{9}$, capillary zone electrophoresis ${ }^{10}$, liquid chromatography-tandem mass spectrometry(LC-MS/MS $)^{11}$ etc. Some of these reported methods suffer from disadvantages such as complicated procedure, long analysis time, expensive instrumentation and relatively low selectivity or sensitivity. Based on its electroactivity, ranitidine hydrochloride has also been investigated and determined conveniently by electrochemical methods ${ }^{2,12-16}$. To our knowledge, voltammetric determinations of ranitidine hydrochloride at a graphene modified glassy carbon electrode have not been reported yet.<smiles>CN/C(=C\[N+](=O)[O-])NCCSCc1ccc(CN(C)C)o1</smiles>

Fig. 1. Chemical structure of ranitidine hydrochloride

Graphene, a single-atom thick planar sheet of $s p^{2}$-bonded carbon atoms closely packed in a two-dimensional honeycomb crystal lattice, has recently attracted tremendous attention due to its unique nanostructure and extraordinary properties ${ }^{17}$. In particular, graphene is an excellent candidate for electrodes material to prepare electrochemical sensors or biosensors in view of its excellent conductivity, fast electron transportation, electrocatalytic activity, high surface area and low manufacturing $\operatorname{cost}^{18}$. In this study, the electrochemical behaviour of ranitidine hydrochloride at graphene modified glassy carbon electrode was investigated in detail. The results showed that graphene modified glassy carbon electrode enhanced the oxidation peak current of ranitidine hydrochloride as compared to bare glassy carbon electrode. Consequently, a voltammetric method based on graphene modified electrode was developed for the determination of ranitidine hydrochloride in pharmaceutical formulations and biological fluids. This newly proposed method possesses several advantages such as high sensitivity, rapid response, good reproducibility, low cost and simplicity. 


\section{EXPERIMENTAL}

All the electrochemical measurements were performed with a CHI 660A Workstation (CH Instruments, Austin, Texas, USA). A conventional three-electrode system, consisting of a graphene modified glassy carbon electrode (3-mm-diameter) as the working electrode, $\mathrm{ag} / \mathrm{AgCl}$ (saturated $\mathrm{KCl}$ ) reference electrode and a platinum wire counter electrode, was employed. Transmission electron microscopic (TEM) image was obtained at a TecnaiG220 microscope (FEI Co., USA).

Graphene was kindly provided by Department of Chemistry, Tsinghua University, China. Standard ranitidine hydrochloride was obtained from National Institute for the Control of Pharmaceutical and Biological Products, China and its stock solution of $1.0 \times 10^{-3} \mathrm{~mol} / \mathrm{L}$ was prepared in water. The working standard solutions were freshly prepared by serial dilution of the stock solution with $0.1 \mathrm{~mol} / \mathrm{L}$ phosphate buffer solution (PBS, pH 6.9). ranitidine hydrochloride capsule (Shanghai Xudong Haipu Pharmaceutical Co. Ltd., Shanghai, China) was purchased from a local pharmacy. All other reagents were of analytical grade and used as received without further purification. Double-distilled water was used throughout. A $0.1 \mathrm{~mol} /$ L PBS (pH 6.9) served as the supporting electrolyte unless otherwise stated.

Fabrication of graphene modified glassy carbon electrode: Graphene was dispersed in ethanol by ultrasonication for $0.5 \mathrm{~h}$ to form a stable and homogeneous dispersion of 1 $\mathrm{mg} / \mathrm{mL}$. Prior to modification, a glassy carbon electrode was mechanically polished with alumina slurry of different grades to a mirror finish and rinsed in water under sonication for $3 \mathrm{~min}$. Then, $10 \mu \mathrm{L}$ of graphene dispersion was dropped on the glassy carbon electrode surface and dried in air.

Sample preparation: A certain amount of ranitidine hydrochloride powder in capsules was transferred into a 100 $\mathrm{mL}$ flask to be dissolved in water and sonicated for $3 \mathrm{~min}$. After being filtered, drug sample solutions were prepared by diluting with $0.1 \mathrm{~mol} / \mathrm{L} \mathrm{PBS} \mathrm{(pH} \mathrm{6.9).} \mathrm{Human} \mathrm{serum} \mathrm{and} \mathrm{urine}$ samples obtained from healthy volunteers were diluted 50 and 20 times with $0.1 \mathrm{~mol} / \mathrm{L} \mathrm{PBS}$ (pH 6.9), respectively, spiked with known amounts of standard solution of ranitidine hydrochloride and further centrifuged for $5 \mathrm{~min}$ at 4,000 rpm. The clear supernatant of the spiked serum and urine samples were diluted with the supporting electrolyte to the appropriate level for the determination.

Measurement: A desired volume of ranitidine hydrochloride standard or sample solution was pipetted to a $10 \mathrm{~mL}$ electrolytic cell containing 0.1 M PBS (pH 6.9), followed by deaeration with pumping oxygen-free nitrogen for $10 \mathrm{~min}$. An accumulation step was then conducted with stirring of the solution for $3 \mathrm{~min}$ at open-circuit. After a quiescent interval of $30 \mathrm{~s}$, linear sweep voltammograms from $0.40-1.20 \mathrm{~V}$ were recorded.

\section{RESULTS AND DISCUSSION}

Characterization of graphene dispersed in ethanol: The TEM of graphene-ethanol dispersion was shown in Fig. 2. It is clear that the transparent, wrinkled and flake-like graphene sheet is well dispersed in ethanol with no aggregation. This wrinkled nature of graphene is highly beneficial in maintaining a high surface area on the electrode since the sheets cannot readily collapse back to a graphitic structure ${ }^{19}$. According to the Randles-Sevcik equation ${ }^{20}$, the electroactive surface areas of bare glassy carbon electrode and graphene modified glassy carbon electrode were measured to be 0.049 and $0.092 \mathrm{~cm}^{2}$, respectively. The results indicated that the electrode effective surface area was increased obviously after modified with graphene.

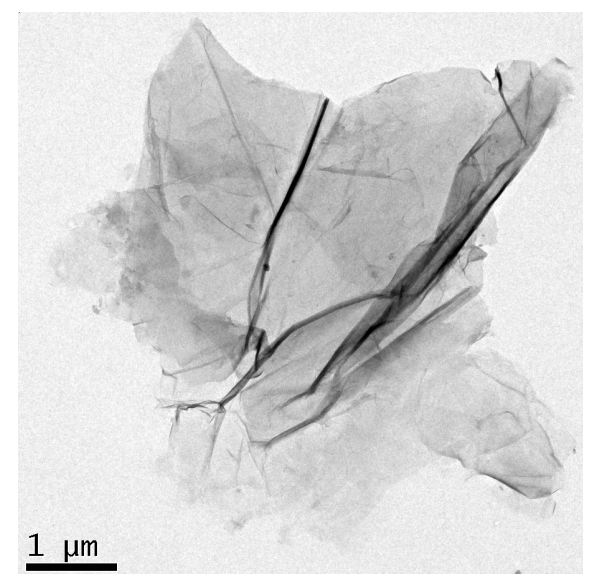

Fig. 2. TEM image of graphene dispersed in ethanol

Electrochemical behaviour of ranitidine hydrochloride: The electrochemical behavior of ranitidine hydrochloride was examined by cyclic voltammetry (CV). As can be seen, no redox peaks were observed at bare glassy carbon electrode (Fig. 3a) and graphene modified glassy carbon electrode (Fig. $3 c$ ) in the blank PBS, indicating that graphene is nonelectroactive in the selected potential window. However, the background of graphene modified glassy carbon electrode was larger than that of bare glassy carbon electrode, which can be attributed to the large specific area and good conductivity of graphene. When $2.0 \times 10^{-5} \mathrm{~mol} / \mathrm{L}$ ranitidine hydrochloride was added into PBS, an ill-defined oxidation peak with low current at about $0.93 \mathrm{~V}$ was observed at bare glassy carbon electrode (Fig. 3b). However, under the same conditions, a sensitive, well-defined oxidation peak of ranitidine hydrochloride with current response of approximately 40 times larger than that at bare glassy carbon electrode was obtained at graphene modified glassy carbon electrode (Fig. 3d). The remarkable peak current enhancement is undoubtedly attributed to the unusual structure and properties of graphene such as high aspect ratio and excellent electrocatalytic activity. Fig. 3 showed that no corresponding reduction peak was observed on the reverse scan, implying that the electrochemical reaction of ranitidine hydrochloride was totally irreversible. The oxidation peak current of ranitidine hydrochloride dramatically decreased during the successive cyclic voltammetric sweeps. After four potential sweeps with a scan rate of $100 \mathrm{mV} / \mathrm{s}$, the peak current remained nearly unchanged. This phenomenon might be ascribed to the adsorption of ranitidine hydrochloride or its oxidation product onto the surface of graphene modified glassy carbon electrode and the resultant inactivation of the electrode surface. The effect of scan rate on the peak current of ranitidine hydrochloride was also investigated by cyclic voltammetry. It was found that the peak current was proportional to the scan 


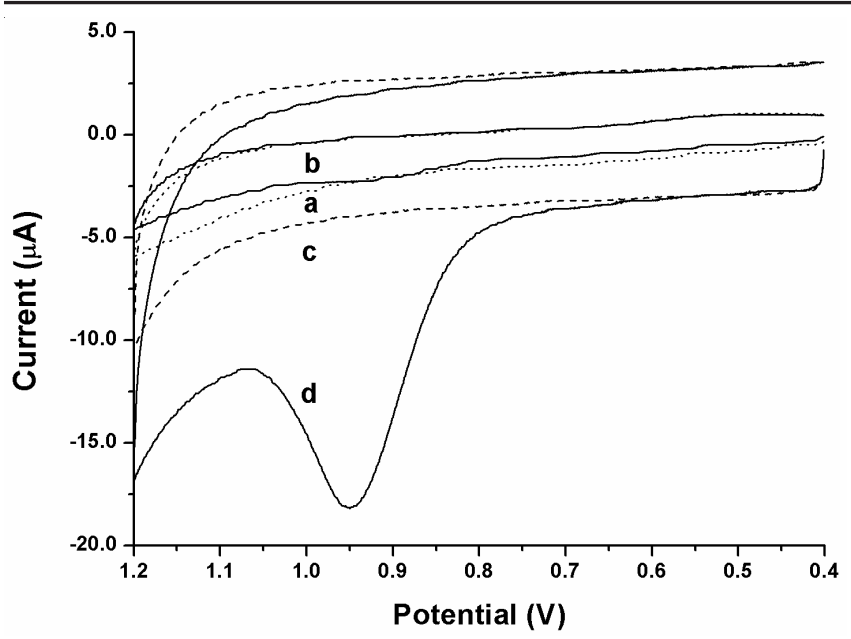

Fig. 3. Cyclic voltammograms of bare glassy carbon electrode (a, b) or graphene modified glassy carbon electrode $(\mathrm{c}, \mathrm{d})$ when placed in $0.1 \mathrm{~mol} / \mathrm{L} \mathrm{PBS} \mathrm{(pH} \mathrm{6.9)} \mathrm{in} \mathrm{the} \mathrm{presence} \mathrm{(b,} \mathrm{d)} \mathrm{and} \mathrm{absence} \mathrm{(a,} \mathrm{c)} \mathrm{of}$ $2.0 \times 10^{-5} \mathrm{~mol} / \mathrm{L}$ ranitidine hydrochloride. Scan rate: $100 \mathrm{mV} / \mathrm{s}$

rate over the range from 10 to $200 \mathrm{mV} / \mathrm{s}$, providing further evidence that the electrode reaction of ranitidine hydrochloride at graphene modified glassy carbon electrode is adsorptioncontrolled.

Effect of supporting electrolyte: The electrochemical oxidation of $2.0 \times 10^{-5} \mathrm{~mol} / \mathrm{L}$ ranitidine hydrochloride in different supporting electrolyte solutions, including $\mathrm{pH}$ 5.0-8.0 PBS, pH 2.0-10.0 Britton-Robinson buffer, $\mathrm{pH}$ 1.0-5.0 sodium citrate- $\mathrm{HCl}$ buffer, $\mathrm{pH} 4.0-6.0 \mathrm{CH}_{3} \mathrm{COOH}-\mathrm{CH}_{3} \mathrm{COONa}$ buffer $(0.1 \mathrm{~mol} / \mathrm{L}$ of each buffer), were examined by cyclic voltammetry. The best oxidation response was obtained in $\mathrm{pH}$ 6.9 PBS in that the electrochemical response was well defined with the highest peak current as compared to that in other buffer systems. Hence, $0.1 \mathrm{~mol} / \mathrm{L}$ PBS ( $\mathrm{pH}$ 6.9) was chosen as the supporting electrolyte for the determination of ranitidine hydrochloride.

Effect of accumulation potential and accumulation time: Accumulation prior to voltammetric measurements could influence the electrooxidation of ranitidine hydrochloride at graphene modified glassy carbon electrode. It was found that the peak current was practically independent on the accumulation potential within the potential window of 0.40 to -0.40 $\mathrm{V}$. Thus, an open circuit accumulation was performed. However, the accumulation time had a remarkable effect on peak current. The peak current increased with the accumulation time in the range of $3 \mathrm{~min}$, when the accumulation time exceeded $3 \mathrm{~min}$ the current reached a plateau, suggesting that the accumulation process of ranitidine hydrochloride had achieved its saturation adsorption on graphene film. Therefore, 3-min accumulation was used to improve sensitivity of the method and shorten the time consumption.

Calibration plot and stability: Under the optimized experimental conditions, the linear sweep voltammograms of ranitidine hydrochloride with different concentrations at graphene modified glassy carbon electrode were recorded. The peak current increased linearly with incremental concentration of ranitidine hydrochloride in the range from $3.0 \times 10^{-7}-1.0 \times$ $10^{-4} \mathrm{~mol} / \mathrm{L}$, giving a regression equation of $\mathrm{i}_{\mathrm{p}}(\mu \mathrm{A})=6.22+$ $4.13 \times 10^{5} \mathrm{C}(\mathrm{mol} / \mathrm{L})(\mathrm{r}=0.9978)$. The detection limit was found to be $1.0 \times 10^{-7} \mathrm{~mol} / \mathrm{L}$ (according to $\mathrm{S} / \mathrm{N}=3$ ). The relative standard deviation (RSD) of $2.6 \%$ for $2.0 \times 10^{-5} \mathrm{~mol} / \mathrm{L}$ ranitidine hydrochloride $(\mathrm{n}=10)$ indicated a good reproducibility.

For evaluating the long-term stability of graphene modified glassy carbon electrode, it was stored in the air and used for monitoring $2.0 \times 10^{-5} \mathrm{~mol} / \mathrm{L}$ ranitidine hydrochloride daily over a period of 4 weeks. The deviation of current responses was only $5.9 \%$.

Interference study: A systematic study was carried out to evaluate the interferences of foreign species on the determination of ranitidine hydrochloride at the level of $1.0 \times 10^{-5}$ $\mathrm{mol} / \mathrm{L}$. We found that 100 -fold concentration of $\mathrm{Na}^{+}, \mathrm{K}^{+}, \mathrm{Mg}^{2+}$, $\mathrm{Pb}^{2+}, \mathrm{Ca}^{2+}, \mathrm{Al}^{3+}, \mathrm{Fe}^{3+}, \mathrm{Cl}^{-}, \mathrm{Br}^{-}, \mathrm{I}^{-}, \mathrm{NO}_{3}{ }^{-}$or $\mathrm{SO}_{4}{ }^{2-}, 20$-fold concentration of uric acid, oxalic acid, citric acid, lactic acid, tartaric acid, glucose, lactose, saccharose, starch, or carboxymethylcellulose and 10-fold concentration of vitamin $B_{1}$, vitamin $B_{2}$, vitamin $B_{12}$ or vitamin $C$ in the solution had almost no influences on the determination of ranitidine hydrochloride (signal change below $5 \%$ ).

Analytical applications: The developed method was used for the determination of ranitidine hydrochloride in capsules (Table-1). Furthermore, the results obtained by present method were compared with those determined by the pharmacopeia $\operatorname{method}^{3}$. The two method results were in good agreement with each other, suggesting that graphene modified glassy carbon electrode had great promise for practical application in pharmaceutical formulation analysis. In order to check the validity of the proposed method, the standard addition method was applied and the recoveries were found to be in the range of 94.5 and $105.8 \%$, indicating that the proposed method had good accuracy and satisfactory repeatability.

\section{TABLE-1}

DETERMINATION OF RANITIDINE HYDROCHLORIDE IN CAPSULES (PERCENTAGE OF THE DECLARED CONTENT, \%)

\begin{tabular}{cccc}
\hline Sample & $\begin{array}{c}\text { Detected content } \\
\text { by this method } \\
(\%)\end{array}$ & $\begin{array}{c}\text { Detected content by } \\
\text { the pharmacopeia } \\
\text { method [Ref. 3] }(\%)\end{array}$ & $\begin{array}{c}\text { Recovery of } \\
\text { this method } \\
(\%)\end{array}$ \\
\hline 1 & 97.8 & 98.4 & 94.5 \\
2 & 99.2 & 99.6 & 101.3 \\
3 & 101.6 & 100.5 & 98.6 \\
4 & 103.8 & 102.9 & 105.8 \\
5 & 98.5 & 99.2 & 97.4 \\
\hline
\end{tabular}

The proposed method was also applied successfully for the determination of ranitidine hydrochloride in spiked human serum and urine samples. The results obtained are summarized in Table-2.

\begin{tabular}{ccccc}
\multicolumn{5}{c}{ TABLE-2 } \\
\multicolumn{5}{c}{$\begin{array}{c}\text { DETERMINATION OF RANITIDINE HYDROCHLORIDE IN } \\
\text { SPIKED HUMAN }\end{array}$} \\
\hline \multirow{2}{*}{ Sample } & $\begin{array}{c}\text { Added } \\
(\mu \mathrm{mol} / \mathrm{L})\end{array}$ & $\begin{array}{c}\text { Found } \\
(\mu \mathrm{mol} / \mathrm{L})\end{array}$ & $\begin{array}{c}\text { Recovery } \\
(\%)\end{array}$ & $\begin{array}{c}\text { RSD } \\
(\%)\end{array}$ \\
\hline Serum 1 & 3.0 & 2.8 & 93.3 & 2.6 \\
2 & 5.0 & 5.2 & 104.0 & 2.8 \\
3 & 8.0 & 8.3 & 103.8 & 2.4 \\
Urine 1 & 3.0 & 3.2 & 106.7 & 2.9 \\
2 & 5.0 & 4.9 & 98.0 & 3.4 \\
3 & 8.0 & 7.8 & 97.5 & 3.8 \\
\hline *Average of five assays. & & &
\end{tabular}

Average of five assays. 


\section{Conclusion}

In this study, a graphene-based electrochemical sensor was fabricated for the voltammetric determination of ranitidine hydrochloride. The enhancement in the oxidation current of ranitidine hydrochloride at graphene modified glassy carbon electrode might be attributed to the unusual structure and properties of graphene. This newly developed method is sensitive, convenient, rapid and suitable for the determination of ranitidine hydrochloride in pharmaceutical formulations and biological fluids.

\section{ACKNOWLEDGEMENTS}

The authors are grateful to the financial support from Applied Scientific and Technological Research Project of Nantong, Jiangsu, China (No. K2006018) and Natural Scientific Research Project of Nantong University (No.10Z015).

\section{REFERENCES}

1. A.S. Amin, I.S. Ahmed, H.A. Dessouki and E.A. Gouda, Spectrochim Acta, A, 59, 695 (2003).

2. W.R. de Araújo and T.R.L.C. Paixão, Electroanalysis, 23, 2549 (2011).

3. Editorial Committee of Pharmacopoeia of People's Republic of China, Pharmacopoeia of People's Republic of China (Part II), China Medical Science Press, Beijing (2010).

4. S.T. Ulu and M. Tuncel, J. Chromatogr. Sci., 50, 301 (2012).
5. M.S. Charde, S.G. Walode, M.R. Tajne and A.V. Kasture, Asian J. Chem., 17, 2402 (2005).

6. Y.X. Chang, Y.Q. Qiu, L.M. Du, C.F. Li and M. Guo, Analyst, 136, 4168 (2011).

7. L.H. Marcolino-Junior, L.C.S. Figueiredo-Filho, H.J. Vieira and O. Fatibello-Filho, Curr. Anal. Chem., 5, 213 (2009).

8. K. Basavaiah and B.C. Somashekar, J. Iran. Chem. Soc., 4, 78 (2007).

9. Y.H. Tang, N.N. Wang, X.Y. Xiong, F.M. Xiong and S.J. Sun, Luminescence, 22, 343 (2007).

10. J.J.B. Nevado, G.C. Peñalvo and R.M.R. Dorado, Anal. Sci., 27, 427 (2011).

11. C. Zhang, L. Wang, X. Guan, J.P. Fawcett, L.M. Zhao, Y.T. Sun and J.K. Gu, Chem. Res. Chin. Univ., 26, 910 (2010).

12. E.Y.Z. Frag, A.M.K. Mohamed, G.G. Mohamed and E.E. Alrahmony, Int. J. Electrochem. Sci., 6, 3508 (2011).

13. K. Vediappan and C.W. Lee, Curr. Appl. Phys., 11, 995 (2011).

14. V. Pfaffen and P.I. Ortiz, Ind. Eng. Chem. Res., 49, 4026 (2010).

15. A. Salimi, M. Izadi, R. Hallaj and M. Rashidi, Electroanalysis, 19, 1668 (2007).

16. P. Norouzi, M.R. Ganjali and P. Daneshgar, J. Pharmacol. Toxicol. Methods, 55, 289 (2007).

17. K.S. Novoselov, A.K. Geim, S.V. Morozov, D. Jiang, Y. Zhang, S.V. Dubonos, I.V. Grigorieva and AA. Firsov, Science, 306, 666 (2004).

18. M. Zhou, Y.M. Zhai and S.J. Dong, Anal. Chem., 81, 5603 (2009).

19. H.C. Schniepp, J.L. Li, M.J. McAllister, H. Sai, M. Herrera-Alonso, D.H. Adamson, R.K. Prud'homme, R. Car, D.A. Saville and I.A. Aksay, J. Phys. Chem. B, 110, 8535 (2006).

20. A.J. Bard and L.R. Faulkner, Electrochemical Methods, Fundamentals and Applications, Wiley, New York (2001). 